\title{
Variações florísticas e estruturais em fragmento de Cerrado após três anos de avaliação ${ }^{1}$
}

\author{
Patrícia Oliveira da Silva ${ }^{2}$; Gisele Cristina de Oliveira Menino ${ }^{3}$; Laísa Cristine dos Santos Silva ${ }^{4}$; \\ Daiane Moreira Dourado ${ }^{5}$; Suzanne da Costa Ribeiro ${ }^{6}$; Thais Cristina Sousa de Oliveira ${ }^{7}$
}

Resumo: Monitoramentos temporais da biodiversidade vegetal são cada vez mais necessários. Diante do exposto, este estudo buscou avaliar se há alterações florísticas e estruturais em fragmento de Cerradão, município de Rio Verde, estado de Goiás, Brasil, em intervalo de tempo de três anos. Foram realizados dois inventários: o primeiro foi realizado no ano de 2011 , onde foram estabelecidas 20 parcelas de $100 \mathrm{~m}^{2}$ cada; o segundo inventário foi realizado em 2014 , onde todos os indivíduos do primeiro levantamento foram remedidos e, aqueles que atingiram os critérios mínimos de inclusão, foram registrados. As alterações ocorridas foram avaliadas por comparações entre: área basal, famílias, gêneros e espécies, número de indivíduos, densidade, dominância, frequência, valor de importância, equabilidade de Pielou e diversidade de Shannon. Foram registradas poucas alterações, e estatisticamente, não houve diferença entre os inventários. Foi excluída a família Calophyllaceae e incluída Burseraceae e, registrou-se a exclusão de seis gêneros e inclusão de três. Em 2011, foram inventariadas 64 espécies, enquanto que em 2014 foram registradas 61. Somaram-se 43 recrutas, enquanto que o número de indivíduos mortos foi de 81. Sendo assim, o total de indivíduos amostrados no primeiro inventário $(\mathrm{n}=394)$ foi superior ao segundo $(n=356)$. Para deteç̧ão de mudanças quanto ao recrutamento e mortalidade de espécies neste ecossistema, recomenda-se que seja estendido o período de monitoramento, com a realização de maior número de inventários.

Palavras - chave: Levantamento florístico; Fitossociologia; Cerradão

\section{Floristic and structural variations in Cerrado fragment after three years of evaluation}

\begin{abstract}
Temporal monitoring of plant biodiversity is increasingly needed. In view of the above, this study sought to evaluate if there are floristic and structural changes in "Cerradão" fragment, Rio Verde city, Goiás state, Brazil, in a time span of three years. Two inventories were carried out: the first one was carried out in 2011, where 20 plots of $100 \mathrm{~m}^{2}$ each were established; the second inventory was performed in 2014, where all individuals from the first inventory were remediated and those who met the minimum inclusion criteria were recorded. The changes were evaluated by comparisons between: basal area, families, genera and species, number of individuals, density, dominance, frequency, importance value, Pielou equability and Shannon diversity. There were few changes, and statistically, there was no difference between inventories. The family Calophyllaceae and included Burseraceae were excluded. Six genera were excluded and three were excluded. In 2011, 64 were inventoried, while in 2014 there were 61 species. The recruits numbered 43, while the number of individuals mortality was 81 . Thus, the total number of individuals sampled in the first inventory $(n=394)$ was higher than the second $(n=356)$. To detect changes in the species recruitment and mortality in this ecosystem, it is recommended that the monitoring period be extended, with a larger number of inventories.
\end{abstract}

Keywords: Floristic survey; Phytosociology; Cerradão

\footnotetext{
${ }^{1}$ Recebido em 01.04.2019 e aceito para publicação como artigo científico em 29.05.2019.

${ }^{2}$ Bióloga, M.Sc. Doutoranda em Ciências Agrárias-Agronomia, Instituto Federal Goiano, campus Rio Verde, Goiás, Brasil. E-mail: <patriciasilvaifgoiano@gmail.com>

${ }_{3}^{3}$ Bióloga, Dra. Professora do Instituto Federal Goiano, campus Rio Verde, Goiás, Brasil. E-mail: <gisele.menino@ifgoiano.edu.br>

4 Bióloga. Mestre em Ciências Agrárias-Agronomia, Instituto Federal Goiano, campus Rio Verde, Goiás, Brasil. E-mail: <laisacristine06@hotmail.com>

${ }^{5}$ Bióloga. Mestranda em Biodiversidade e Conservação, Instituto Federal Goiano, campus Rio Verde, Goiás, Brasil. E-mail: <douradobio@hotmail.com>

${ }^{6}$ Bióloga. Instituto Federal Goiano, campus Rio Verde, Goiás, Brasil. E-mail: <suzanne.ribbeiro@gmail.com>

${ }^{7}$ Bióloga. Mestranda em Biodiversidade e Conservação, Instituto Federal Goiano, campus Rio Verde, Goiás, Brasil. E-mail: <thaiscristinasousasab@gmail.com>
} 


\section{Introdução}

A análise florística e estrutural da comunidade arbórea de ecossistemas restaurados, ou em processos de restauração, é uma indicadora eficiente para impulsionar os conhecimentos ecológicos desses ambientes, avaliar e monitorar as fases de sucessões do processo de restauração da vegetação, bem como promover avanços em pesquisas subsequentes (DARONCO et al., 2013). Estes estudos têm sido realizados frequentemente, mas não na savana tropical mais rica do mundo, o Cerrado (CARVALHO et al., 2016). Se tratando da vegetação deste bioma, as fisionomias que mais se destacam é o Cerradão e o Cerrado sensu stricto, sendo ambas muito requeridas para fins de conversão agropecuária, devido as suas características de relevo e solos que permitem à mecanização agrícola intensiva (CARVALHO et al., 2016).

O Cerradão corresponde a uma "floresta mesófila esclerófila", que se caracteriza por um sub-bosque formado por pequenos arbustos e ervas, com poucas gramíneas (CARVALHO et al., 2016). Esse ecossistema é composto pela presença preferencial de espécies que ocorrem no cerrado sentido restrito e também por espécies de florestas, particularmente as de Mata Seca Semidecídua e da Mata de Galeria não-inundável. Do ponto de vista fisionômico é uma floresta, mas floristicamente se assemelha mais ao Cerrado sensu stricto (PRADOJÚNIOR et al., 2012). E, assim como qualquer outra fisionomia do Cerrado, o Cerradão está em constantes alterações, sejam florísticas ou fitossociológicas. Entretanto, estudos que abordam essas mudanças nesta mesma fisionomia ainda são escassos.

Monitoramentos temporais da biodiversidade que avaliam o comportamento da comunidade quanto à estabilidade, levam em consideração dados demográficos, composição de espécies, turnover, recrutamento, crescimento, mortalidade, biologia das espécies e produção de biomassa, fornecem informações sobre o funcionamento das comunidades em longo prazo e a resposta das mesmas aos distúrbios ocasionais (LIBANO e FELFILI, 2006). Além disso, a caracterização do recrutamento e da mortalidade permite concluir sobre a facilidade ou dificuldade de uma espécie em colonizar determinado ambiente, os processos responsáveis pela flutuação destas taxas na população (WATKINSON, 1997), a identificação da capacidade de regeneração e da ocorrência de perturbações em determinado local (HARPER, 1977).

Dentre as variáveis que podem causar distúrbios ou perturbações às comunidades vegetais estão os efeitos da fragmentação, ações antrópicas e flutuações climáticas. Esses fatores têm influenciado o funcionamento dos ecossistemas, alterando sua regeneração natural e conduzindo as comunidades vegetais às dinâmicas desconhecidas (O'BRIEN et al., 2012). Sendo assim, os ciclos florestais podem estar mais curtos e as comunidades mais dinâmicas (LAURANCE et al., 2009), pois as áreas de ocorrência das espécies estão sendo alteradas (FEELEY et al., 2011). Com a finalidade de captar tais flutuações, nos ambientes de Cerrado os monitoramentos de longo prazo devem ser realizados, inicialmente, com mensurações regulares em curtos intervalos de tempo, de no mínimo três anos, pois em intervalos mais amplos as oscilações se diluem no tempo e deixam de ser perceptíveis (FELFILI et al., 2000).

Diante do exposto, há a necessidade de se realizar monitoramentos temporais em remanescentes do Cerrado e, este estudo buscou avaliar se há e quais são as alterações/mudanças florísticas e estruturais ocorridas em fragmento de Cerradão, localizado no Estado de Goiás, Município de Rio Verde, em intervalo de tempo de três anos.

\section{Material e métodos}

Área de estudo

O estudo foi realizado em remanescente de Cerrado do Instituto Federal Goiano (1749'0,40" S; 5053'58,57" O), no município de Rio Verde, região sudoeste do estado de 
Goiás, Brasil. O fragmento ocupa uma extensão total de 26,3 ha e é caracterizado fisionomicamente como Cerradão. A área é circundada por plantios experimentais de grandes culturas e margeada pela rodovia Sul Goiana. A classificação climática para a região, segundo Köppen, é do tipo Aw (tropical típico), alternadamente úmido (de outubro a abril) e seco (de maio a setembro), com temperatura média do mês mais frio superior a $18{ }^{\circ} \mathrm{C}$ e precipitação pluviométrica anual inferior a $2.000 \mathrm{~mm}$ por ano.

Coletas e análises dos dados

Para análise florística e fitossociológica da área de estudo foram realizados dois inventários: o primeiro no ano de 2011, onde foram estabelecidas 20 parcelas sistematizadas de $10 \mathrm{~m} \times 10 \mathrm{~ms}\left(100 \mathrm{~m}^{2}\right.$ cada $)$, distanciadas em $10 \mathrm{~m}$ entre si e distribuídas em quatro transectos (cinco parcelas por transecto), totalizando uma área amostral de 0,2 ha. As parcelas foram distribuídas paralelamente à borda em direção ao interior do fragmento, sendo a distância total do primeiro para o último transecto de $30 \mathrm{~m}$. Todos os indivíduos arbóreos-arbustivos com CAP (Circunferência à Altura do Peito medida à altura de $1,30 \mathrm{~cm}$ em relação ao nível do solo) $\geq 10 \mathrm{~cm}$ receberam etiquetas de alumínio numeradas, e foram mensurados o CAP com auxílio de uma fita métrica. Para avaliar as alterações ocorridas em intervalo de tempo de três anos, um segundo inventário foi realizado no ano de 2014 , onde todos os indivíduos do primeiro levantamento foram remedidos e, aqueles que atingiram os critérios mínimos de inclusão ( $\geq 10 \mathrm{~cm}$ de CAP a $1,30 \mathrm{~cm}$ do solo), foram registrados $\mathrm{e}$ denominados de recrutas.

Todos os indivíduos foram identificados ao nível de família, gênero e espécies in loco e, quando necessário, por meio de comparações com literatura especializada ou exsicatas do Herbário do Instituto Federal Goiano - Campus Rio Verde e envio a especialistas. A nomenclatura botânica foi conferida segundo Missouri Botanical Garden (disponível em: http://www.tropicos.org).

As alterações ocorridas na área estudada foram avaliadas por meio de comparações entre os parâmetros de área basal, número de famílias, gêneros e espécies, número de indivíduos, pelos parâmetros fitossociológicos (MUELLER-DOMBOIS e ELENBERG, 1974) relativos de densidade, dominância, frequência e obtidos a partir desses, o valor de importância (VI) de cada espécie amostrada e ainda pelos índices de equabilidade de Pielou (J') (PIELOU, 1966) e índice de diversidade de Shannon (H') (SHANNON, 1948). Além disso, também se realizou a distribuição em classes diamétricas.

Para comparar as classes diamétricas, densidade, índice de diversidade de Shannon e equabilidade de Pielou, entre os dois levantamentos, utilizou-se o teste " $t$ " de Student (SOKAL e ROHLF, 1984), e para comparar a área basal entre os inventários, utilizou-se o teste do Qui-quadrado (ZAR, 1998).

\section{Resultados}

A comunidade arbustiva-arbórea no fragmento de Cerradão apresentou poucas alterações florísticas e estruturais em um intervalo de tempo de três anos (2011-2014) (Tabela 1). De modo geral, não houve diferença entre os levantamentos para nenhuma das variáveis estudadas. Em termos de dinâmica de recrutamento, a família Calophyllaceae foi excluída e houve inclusão de Burseraceae; seis gêneros foram excluídos (Bauhinia, Buchenavia, Kielmeyera, Magonia, Psidium e Tocoyena) e houve inclusão de apenas três (Anadenanthera, Cordiera e Protium).

O número de espécies também apresentou variação, onde em 2011 foram inventariadas 64 espécies e em 2014 esse número reduziu para 61 , pois houve a saída de seis espécies e entrada de três. Os recrutas, que são aqueles que atenderam aos mesmos critérios das demais plantas amostradas, somaram-se apenas 43, enquanto que o número de indivíduos mortos foi quase que o dobro dos incrementos (81 
plantas). Sendo assim, o total de indivíduos amostrados no primeiro inventário $(n=394)$ foi superior ao segundo $(n=356)$, no entanto não houve diferença $(p>0,05)$ na densidade entre os levantamentos.

Tabela 1. Comparação florística e estrutural entre dois inventários, com intervalo de três anos (2011 e 2014), em um fragmento de Cerradão, Município de Rio Verde Estado de Goiás.

Table 1. Floristic and structural comparison between two inventories, with interval of three years (2011 and 2014), in a fragment of Cerradão, Rio Verde city, Goiás state.

\begin{tabular}{ccc}
\hline Amostragem & $\mathbf{2 0 1 1}$ & $\mathbf{2 0 1 4}$ \\
\hline Área amostrada & $0,2 \mathrm{ha}$ & $0,2 \mathrm{ha}$ \\
Famílias & 28 & 28 \\
Gênero & 50 & 47 \\
Espécies & 64 & 61 \\
Recrutas & - & 43 \\
Mortos & - & 81 \\
Indivíduos & 394 & 356 \\
Índice de Shannon (H') & 3,29 & 3,20 \\
Equabilidade de Pielou (J') & 0,79 & 0,78 \\
Área basal & $2,62 \mathrm{~m}^{2} \mathrm{ha}^{-1}$ & $2,64 \mathrm{~m}^{2} \mathrm{ha}^{-1}$ \\
\hline
\end{tabular}

Em termos de diversidade, o fragmento também apresentou poucas alterações, sendo assim as diferenças dos índices entre os anos de levantamento não foram significativas $(\mathrm{p}>0,05)$. Em um intervalo de três anos houve redução no índice de diversidade de Shannon e na Equabilidade de Pielou; já a área basal apresentou comportamento inverso, mas não o suficiente para promover diferenças $(p>0,05)$ entre os inventários.

No fragmento de Cerradão a família mais representativa foi Sapotaceae, embora com única espécie, apresentou 81 e 75 indivíduos para 2011 e 2014, respectivamente. A família com maior número de espécies foi Fabaceae (n =11) e, apesar de haver a saída de uma espécie (Bauhinia cheilantha), houve a entrada de outra (Anadenanthera colubrina (Vell.) Brenan), em 2014, mantendo assim o número de espécies para tal família. Além de A. colubrina, ainda houve o registro de duas outras espécies, que são a Cordiera sessilis (Vell.) Kuntze, representante da família Rubiaceae, e Protium spruceanum (Benth.) Engl., representando a nova família recrutada, Burseraceae. A lista florística completa, assim como as alterações na estrutura das espécies de ocorrência, tanto em 2011 quanto em 2014, estão representadas na Tabela 2.

Em consequência da maior representatividade de indivíduos da família Sapotaceae, registrou-se também a maior densidade, dominância, frequência e valor de importância (VI) para a única espécie que a representou neste estudo, Chrysophyllum marginatum.

As 10 espécies com maior VI para o ano de 2011 foram C. marginatum, Averrhoidium gardnerianum, Myracrodruon urundeuva, Terminalia argentea, Xylopia aromatica, Qualea parviflora, Lithrea molleoides, Emmotum nitens, Qualea grandiflora e Tachigali paniculata; Já no de 2014, foram as mesmas espécies com maior VI, porém em ordem diferente para as últimas colocações: $C$. marginatum, A. gardnerianum, M. urundeuva, T. argentea, $X$. aromatica, L. molleoides, $Q$. grandiflora, $Q$. parviflora, $T$. paniculata e $E$. nitens. 
Tabela 2. Alterações florísticas e estruturais após três anos (2011 e 2014) de monitoramento, em um fragmento de Cerradão, Município de Rio Verde, Goiás.

Table 2. Structural and floristic changes after three years (2011 and 2014) monitoring in a fragment of Cerradão, Rio Verde city, Goiás state.

\begin{tabular}{|c|c|c|c|c|c|c|c|c|}
\hline \multirow{2}{*}{$\begin{array}{c}\text { FAMÍLIA/Espécies } \\
\text { ANACARDIACEAE }\end{array}$} & \multicolumn{2}{|c|}{ DR } & \multicolumn{2}{|c|}{ DoR } & \multicolumn{2}{|c|}{ FR } & \multicolumn{2}{|c|}{ VI } \\
\hline & 2011 & 2014 & 2011 & 2014 & 2011 & 2014 & 2011 & 2014 \\
\hline Lithraea molleoides (Vell.) Engl. & 4,09 & 3,37 & 3,57 & 4,06 & 4,19 & 4,5 & 11,84 & 11,93 \\
\hline Myracrodruon urundeuva Allemão & 7,42 & 8,15 & 4,57 & 7,63 & 5,58 & 6,0 & 17,57 & 21,78 \\
\hline \multicolumn{9}{|l|}{ ANNONACEAE } \\
\hline Annona cacans Warm. & 0,51 & 0,56 & 0,28 & 0,77 & 0,93 & 1,0 & 1,73 & 2,33 \\
\hline Annona sp. & 0,26 & 0,28 & 0,27 & 0,25 & 0,47 & 0,5 & 0,99 & 1,03 \\
\hline Cardiopetalum calophyllum Schltdl. & 1,02 & 2,25 & 0,60 & 0,78 & 1,86 & 3,0 & 3,48 & 6,02 \\
\hline Xylopia aromatica (Lam.) Mart. & 4,35 & 3,93 & 7,08 & 6,31 & 3,26 & 4,0 & 14,69 & 14,24 \\
\hline \multicolumn{9}{|l|}{ APOCYNACEAE } \\
\hline Aspidosperma macrocarpum Mart. & 0,26 & 0,84 & 0,04 & 0,12 & 0,47 & 1,0 & 0,76 & 1,97 \\
\hline Aspidosperma tomentosum Mart. & 0,51 & 0,56 & 0,21 & 0,29 & 0,93 & 1,0 & 1,65 & 1,85 \\
\hline \multicolumn{9}{|l|}{ BIGNONIACEAE } \\
\hline Cybistax antisyphilitica (Mart.) Mart. & 0,51 & 0,56 & 0,22 & 0,28 & 0,93 & 1,0 & 1,66 & 1,84 \\
\hline Handroanthus aureus Mattos & 0,26 & 0,28 & 0,56 & 0,61 & 0,47 & 0,5 & 1,28 & 1,39 \\
\hline \multicolumn{9}{|l|}{ BORAGINACEAE } \\
\hline Cordia glabrata (Mart.) A. DC. & 0,26 & 0,28 & 0,06 & 0,1 & 0,47 & 0,5 & 0,78 & 0,88 \\
\hline \multicolumn{9}{|l|}{ BURSERACEAE } \\
\hline Protium spruceanum (Benth.) Engl. & - & 0,28 & - & 0,05 & - & 0,5 & - & 0,84 \\
\hline \multicolumn{9}{|l|}{ CALOPHYLLACEAE } \\
\hline Kielmeyera sp. & 0,26 & - & 0,20 & - & 0,47 & - & 0,92 & - \\
\hline \multicolumn{9}{|l|}{ CARYOCARACEAE } \\
\hline Caryocar brasiliense Cambess. & 0,77 & 0,56 & 1,56 & 1,43 & 1,40 & 1,0 & 3,72 & 3,00 \\
\hline \multicolumn{9}{|l|}{ COMBRETACEAE } \\
\hline Buchenavia tomentosa Eichler. & 0,26 & - & 0,06 & - & 0,47 & - & 0,78 & - \\
\hline Terminalia argentea Mart. \& Zucc. & 3,32 & 2,53 & 8,90 & 8,41 & 3,72 & 3,5 & 15,95 & 14,43 \\
\hline \multicolumn{9}{|l|}{ DILLENIACEAE } \\
\hline Curatella americana $\mathrm{L}$. & 1,79 & 1,69 & 2,80 & 1,54 & 2,33 & 2,5 & 6,91 & 5,72 \\
\hline \multicolumn{9}{|l|}{ EBENACEAE } \\
\hline Diospyros burchellii Hiern. & 0,51 & 0,56 & 0,38 & 0,5 & 0,93 & 1,0 & 1,83 & 2,06 \\
\hline \multicolumn{9}{|l|}{ ERYTHROXYLACEAE } \\
\hline Erythroxylum suberosum A.St.-Hil. & 0,77 & 0,56 & 0,16 & 0,18 & 0,93 & 0,5 & 1,86 & 1,24 \\
\hline \multicolumn{9}{|l|}{ FABACEAE } \\
\hline Acacia polyphylla DC. & 0,77 & 0,84 & 0,12 & 0,53 & 0,93 & 1,0 & 1,81 & 2,38 \\
\hline Acosmium dasycarpum (Vogel) Yakovlev & 0,51 & 0,28 & 0,13 & 0,16 & 0,93 & 0,5 & 1,57 & 0,94 \\
\hline Anadenanthera colubrina (Vell.) Brenan & - & 0,28 & - & 0,12 & - & 0,5 & - & 0,90 \\
\hline Andira vermifuga Mart. ex Benth. & 0,26 & 0,28 & 0,55 & 0,81 & 0,47 & 0,5 & 1,27 & 1,59 \\
\hline Bauhinia cheilantha (Bong.) Steud. & 0,26 & - & 0,22 & - & 0,47 & - & 0,94 & - \\
\hline Dimorphandra mollis Benth. & 0,26 & 0,28 & 0,15 & 0,13 & 0,47 & 0,5 & 0,88 & 0,91 \\
\hline Lonchocarpus sp. & 0,26 & 0,28 & 1,59 & 2,63 & 0,47 & 0,5 & 2,31 & $\begin{array}{c}3,41 \\
\text { continua }\end{array}$ \\
\hline
\end{tabular}


continuação

Tabela 2. Alterações florísticas e estruturais após três anos (2011 e 2014) de monitoramento, em um fragmento de Cerradão, Município de Rio Verde, Goiás.

Table 2. Structural and floristic changes after three years (2011 and 2014) monitoring in a fragment of Cerradão, Rio Verde city, Goiás state.

\begin{tabular}{|c|c|c|c|c|c|c|c|c|}
\hline \multirow{2}{*}{$\begin{array}{c}\text { FAMÍLIA/Espécies } \\
\text { FABACEAE }\end{array}$} & \multicolumn{2}{|c|}{ DR } & \multicolumn{2}{|c|}{ DoR } & \multicolumn{2}{|c|}{ FR } & \multicolumn{2}{|c|}{ VI } \\
\hline & 2011 & 2014 & 2011 & 2014 & 2011 & 2014 & 2011 & 2014 \\
\hline Machaerium acutifolium Vogel & 1,79 & 2,53 & 0,65 & 0,88 & 2,33 & 3,0 & 4,77 & 6,41 \\
\hline Pterodon emarginatus Vogel & 0,26 & 0,28 & 0,47 & 0,67 & 0,47 & 0,5 & 1,19 & 1,45 \\
\hline Senegalia polyphylla (DC.) Britton & 1,02 & 1,12 & 0,62 & 1,16 & 1,40 & 1,5 & 3,03 & 3,78 \\
\hline Stryphnodendron adstringens (Mart.) Coville & 0,51 & 0,56 & 1,59 & 1,64 & 0,93 & 1,0 & 3,03 & 3,20 \\
\hline Tachigali paniculata Aubl. & 3,07 & 3,65 & 2,75 & 3,08 & 3,26 & 3,5 & 9,07 & 10,23 \\
\hline \multicolumn{9}{|l|}{ LAMIACEAE } \\
\hline $\begin{array}{l}\text { Aegiphila lhotskiana Cham. } \\
\text { LAURACEAE }\end{array}$ & 0,26 & 0,28 & 0,16 & 0,38 & 0,47 & 0,5 & 0,88 & 1,16 \\
\hline Nectandra sp. & 0,26 & 0,28 & 0,37 & 1,12 & 0,47 & 0,5 & 1,09 & 1,90 \\
\hline \multicolumn{9}{|l|}{ MALPIGHIACEAE } \\
\hline Byrsonima basiloba A. Juss. & 0,26 & 0,28 & 0,39 & 0,47 & 0,47 & 0,5 & 1,11 & 1,25 \\
\hline Byrsonima crassa Nied. & 2,81 & 3,65 & 1,04 & 1,91 & 3,26 & 3,5 & 7,11 & 9,07 \\
\hline Byrsonima crassifolia $($ L.) Kunth & 0,26 & - & 0,07 & - & 0,47 & - & 0,79 & - \\
\hline Byrsonima laxiflora Griseb. & 0,51 & 0,28 & 0,12 & 0,05 & 0,47 & 0,5 & 1,10 & 0,83 \\
\hline Byrsonima pachyphylla A. Juss. & 0,26 & 0,84 & 0,35 & 0,44 & 0,47 & 1,5 & 1,07 & 2,79 \\
\hline Byrsonima verbascifolia (L.) DC. & 1,53 & 1,4 & 0,83 & 0,51 & 1,86 & 2,0 & 4,23 & 3,91 \\
\hline \multicolumn{9}{|l|}{ MALVACEAE } \\
\hline Eriotheca candolleana (K. Schum.) A. Robyns & 0,26 & 0,28 & 0,19 & 0,22 & 0,47 & 0,5 & 0,91 & 1,00 \\
\hline $\begin{array}{l}\text { Eriotheca pubescens (Mart. \& Zucc.) Schott. \& } \\
\text { Endl. }\end{array}$ & 1,53 & 1,12 & 2,24 & 1,8 & 2,33 & 1,5 & 6,10 & 4,43 \\
\hline $\begin{array}{l}\text { Luehea grandiflora Mart. \& Zucc. } \\
\text { MELIACEAE }\end{array}$ & 0,51 & 0,56 & 1,07 & 1,05 & 0,93 & 1,0 & 2,51 & 2,61 \\
\hline \multicolumn{8}{|l|}{ METTENIUSACEAE } & 1,87 \\
\hline Emmotum nitens (Benth.) Miers & 4,35 & 3,93 & 3,39 & 2,45 & 3,72 & 3,5 & 11,46 & 9,88 \\
\hline \multicolumn{9}{|l|}{ MORACEAE } \\
\hline \multicolumn{9}{|l|}{ MYRTACEAE } \\
\hline Blepharocalyx salicifolius (Kunth) O. Berg & 0,26 & 0,28 & 0,06 & 0,08 & 0,47 & 0,5 & 0,78 & 0,86 \\
\hline Myrcia splendens (Sw.) DC. & 0,26 & 0,28 & 0,06 & 0,07 & 0,47 & 0,5 & 0,78 & 0,85 \\
\hline Myrcia tomentosa (Aubl.) DC. & 0,26 & 0,56 & 0,13 & 0,17 & 0,47 & 0,5 & 0,85 & 1,23 \\
\hline Psidium sp. & 0,77 & - & 2,38 & - & 0,93 & - & 4,07 & - \\
\hline \multicolumn{9}{|l|}{ PRIMULACEAE } \\
\hline $\begin{array}{l}\text { Myrsine umbellata Mart. } \\
\text { PROTEACEAE }\end{array}$ & 0,51 & 0,28 & 0,22 & 0,05 & 0,47 & 0,5 & 1,19 & 0,83 \\
\hline Roupala montana Aubl. & 3,32 & 3,37 & 2,15 & 1,95 & 3,26 & 3,5 & 8,73 & $\begin{array}{c}8,82 \\
\text { continua }\end{array}$ \\
\hline
\end{tabular}


continuação

Tabela 2. Alterações florísticas e estruturais após três anos (2011 e 2014) de monitoramento, em um fragmento de Cerradão, Município de Rio Verde, Goiás.

Table 2. Structural and floristic changes after three years (2011 and 2014) monitoring in a fragment of Cerradão, Rio Verde city, Goiás state.

\begin{tabular}{|c|c|c|c|c|c|c|c|c|}
\hline \multirow{2}{*}{$\begin{array}{l}\text { FAMÍLIA/Espécies } \\
\text { RHAMNACEAE }\end{array}$} & \multicolumn{2}{|c|}{ DR } & \multicolumn{2}{|c|}{ DoR } & \multicolumn{2}{|c|}{ FR } & \multicolumn{2}{|c|}{ VI } \\
\hline & 2011 & 2014 & 2011 & 2014 & 2011 & 2014 & 2011 & 2014 \\
\hline Rhamnidium elaeocarpum Reissek & 1,28 & 1,12 & 1,95 & 1,88 & 1,86 & 1,5 & 5,09 & 4,50 \\
\hline \multicolumn{9}{|l|}{ RUBIACEAE } \\
\hline Cordiera sessilis (Vell.) Kuntze & - & 0,28 & - & 0,11 & - & 0,5 & - & 0,89 \\
\hline Tocoyena bullata (Vell.) Mart. & 0,51 & - & 0,08 & - & 0,93 & - & 1,52 & - \\
\hline \multicolumn{9}{|l|}{ RUTACEAE } \\
\hline Zanthoxylum monogynum A.St.-Hil. & 0,26 & 0,28 & 0,24 & 0,25 & 0,47 & 0,5 & 0,96 & 1,03 \\
\hline Zanthoxylum rhoifolium Lam. & 0,26 & 0,28 & 0,03 & 0,05 & 0,47 & 0,5 & 0,75 & 0,83 \\
\hline \multicolumn{9}{|l|}{ SAPINDACEAE } \\
\hline Averrhoidium gardnerianum Baill. & 8,95 & 10,96 & 9,06 & 14,3 & 6,51 & 7,5 & 24,52 & 32,75 \\
\hline Dilodendron bipinnatum Radlk. & 0,26 & 0,28 & 1,90 & 2,22 & 0,47 & 0,5 & 2,62 & 3,00 \\
\hline Magonia pubescens A.St.-Hil. & 0,26 & - & 0,12 & - & 0,47 & - & 0,84 & - \\
\hline Talisia esculenta (A.St.-Hil.) Radlk. & 1,79 & 0,56 & 1,63 & 0,91 & 2,79 & 1,0 & 6,21 & 2,47 \\
\hline SAPOTACEAE & & & & & & & & \\
\hline $\begin{array}{l}\text { Chrysophyllum marginatum (Hook. \& Arn.) } \\
\text { Radlk. }\end{array}$ & 20,7 & 21,07 & 18,74 & 15,93 & 8,37 & 9,0 & 47,83 & 45,99 \\
\hline \multicolumn{9}{|l|}{ VOCHYSIACEAE } \\
\hline Callisthene fasciculata Mart. & 0,26 & 0,28 & 0,51 & 0,59 & 0,47 & 0,5 & 1,23 & 1,37 \\
\hline Qualea grandiflora Mart. & 3,32 & 3,37 & 2,26 & 2,77 & 4,19 & 4,5 & 9,77 & 10,64 \\
\hline Qualea multiflora Mart. & 0,51 & 0,56 & 0,27 & 0,1 & 0,93 & 1,0 & 1,71 & 1,66 \\
\hline Qualea parviflora Mart. & 3,84 & 4,21 & 4,23 & 1,65 & 4,19 & 4,5 & 12,25 & 10,36 \\
\hline Indeterminada 1 & 0,26 & 0,28 & 0,28 & 0,29 & 0,47 & 0,5 & 1,00 & 1,07 \\
\hline Indeterminada 2 & 0,26 & 0,28 & 0,77 & 0,78 & 0,47 & 0,5 & 1,49 & 1,56 \\
\hline Espécie sem folhas & 2,05 & - & 1,93 & - & 1,40 & - & 5,37 & - \\
\hline
\end{tabular}

Onde: DR (densidade reativa); DoR (dominância relativa); FR (frequência relativa); VI (valor de importância).

Dentre as 10 espécies com maiores valores de importância (VI), a que apresentou maior área basal $(\mathrm{AB})$, foi consequentemente, a espécie mais abundante na área de estudo, $C$. marginatum, seguido de A. gardnerianum, $M$. urundeuva e $T$. argentea. De todas as espécies registradas, houve incremento em área basal em apenas cinco espécies; a maior redução em área basal ocorreu na espécie $Q$. parviflora, onde houve redução de mais da metade de sua $\mathrm{AB}$ (Tabela 3).

Se tratando da distribuição diamétrica, verificou-se que há mais plantas nas categorias de menor CAP, compondo assim o padrão de Jinvertido para ambos os anos inventariados (Figura 1). Embora, tenha se registrado uma redução dos indivíduos em cada classe diamétrica, não houve diferença $(\mathrm{p}>0,05)$ entre os levantamentos.

A categoria em que se enquadra a maior parte dos indivíduos amostrados neste estudo é a de menor CAP (3-6 cm). Apenas quatro indivíduos compõem a categoria de maior valor de CAP (24-48 cm). Entretanto, de modo geral, 
em função do menor número de indivíduos encontrados no segundo levantamento, as categorias do ano de 2014 também apresentam menor número de indivíduos. É possível observar que a categoria que apresentou maior número de indivíduos mortos (20 plantas) é a classe com os menores valores de CAP.

Tabela 3. Área basal total das 10 espécies com maior índice de valor de importância após três anos (2011 e 2014) de monitoramento, em um fragmento de Cerradão, Município de Rio Verde, Estado de Goiás.

Table 3. Total basal area of the 10 species with the highest importance value index after three years (2011 and 2014) monitoring, in a fragment of Cerradão, Rio Verde city, Goiás state.

\begin{tabular}{|c|c|c|}
\hline \multirow{2}{*}{ Espécies } & \multicolumn{2}{|c|}{ Área Basal (AB) } \\
\hline & \multicolumn{2}{|c|}{$\mathrm{m}^{2} \mathrm{ha}^{-1}$} \\
\hline & 2011 & 2014 \\
\hline Chrysophyllum marginatum (Hook. \& Arn.) Radlk. & 0,49 & 0,42 \\
\hline Averrhoidium gardnerianum Baill. & 0,24 & 0,38 \\
\hline Myracrodruon urundeuva Allemão & 0,12 & 0,20 \\
\hline Terminalia argentea Mart. (Cambess.) Mart. & 0,23 & 0,22 \\
\hline Xylopia aromatica (Lam.) Mart. & 0,19 & 0,17 \\
\hline Qualea parviflora Mart. & 0,11 & 0,04 \\
\hline Lithraea molleoides (Vell.) Engl. & 0,09 & 0,11 \\
\hline Emmotum nitens (Benth.) Miers & 0,09 & 0,06 \\
\hline Qualea grandiflora Mart. & 0,06 & 0,07 \\
\hline Tachigali paniculata Aubl. & 0,07 & 0,08 \\
\hline
\end{tabular}

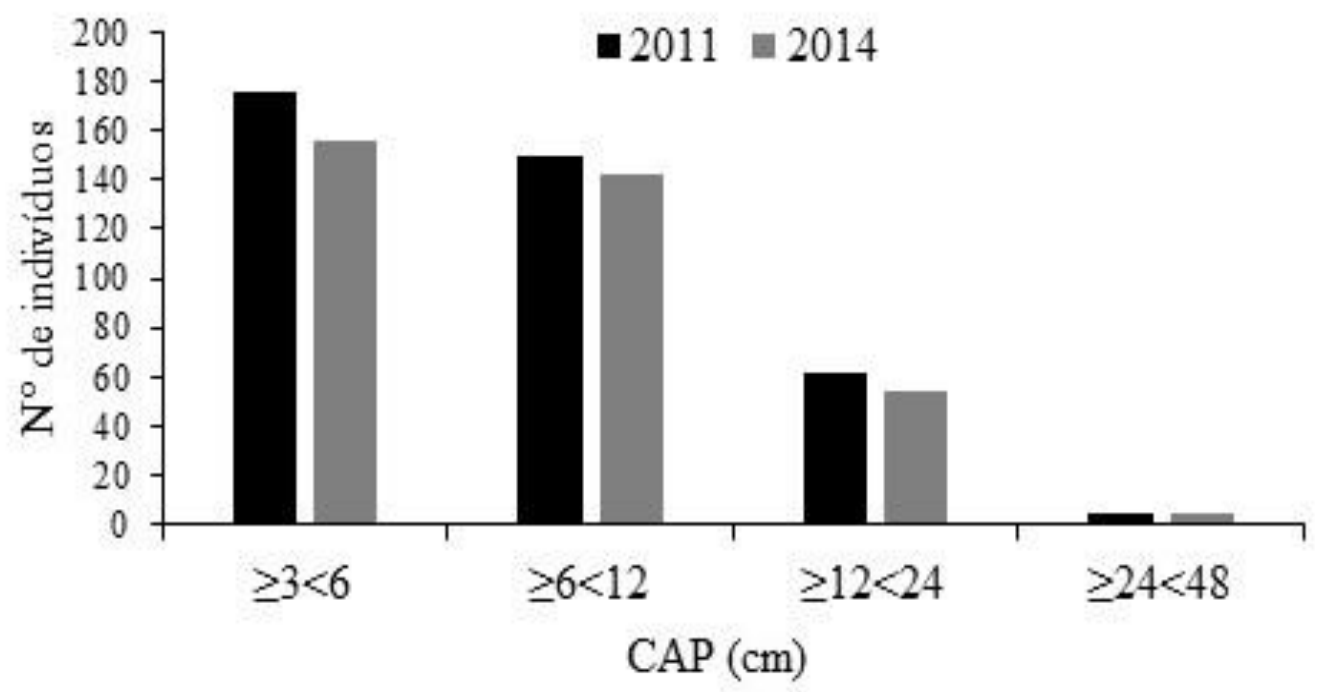

Figura 1. Distribuição diamétrica dos indivíduos arbustivos-arbóreos após três anos (2011 e 2014) de monitoramento, em um fragmento de Cerradão, Município de Rio Verde, Goiás.

Figure 1. Diametric distribution of shrub-tree individuals after three years (2011 and 2014) monitoring in a fragment of Cerradão, Rio Verde city, Goiás. 


\section{Discussão}

No fragmento de Cerradão não houve diferenças para as variáveis fitossociológicas e de diversidade entre os dois inventários, no entanto, as poucas alterações ocorridas após o intervalo de três anos para o Cerrado devem ser levadas em consideração, pois se trata de uma vegetação que está em constante influencia antrópica. Em relação ao recrutamento de espécies, percebe-se que a saída é maior do que a entrada. No entanto, as espécies que desapareceram e as que surgiram nos levantamentos são espécies consideradas pouco abundantes (LIBANO e FELFILI, 2006). Dessa forma, a diversidade de Shannon, mesmo que tenha sofrida uma redução e não tenha apresentado diferença entre os levantamentos, ainda continua alta na área amostrada $\left(\mathrm{H}^{\prime}=3,29\right.$ (2011) e 3,20 (2014)). Esses valores estão de acordo com os observados em outras formações florestais (GIÁCOMO et al., 2015). Diante disso, a riqueza florística e a diversidade da área, ainda que em pequenas proporções, sofreram modificações entre os levantamentos, porém a área tem mantido as características originais da vegetação, sugerindo que é relativamente estável (LIBANO e FELFILI, 2006).

Apesar de não ter constatado diferença entre os inventários, registrou-se uma redução da densidade e, mesmo que muito pouco, um incremento na área basal. A diminuição da densidade ocorreu em função da mortalidade ter sido mais alta do que o recrutamento na comunidade estudada. Entretanto, os indivíduos sobreviventes apresentaram aumento no armazenamento de biomassa durante o intervalo de três anos, causando incremento na área basal, comportamento esse que está de acordo com alguns estudos (DINIZ et al., 2017; REIS et al., 2018).

$\mathrm{Na}$ distribuição diamétrica é possível verificar a maior mortalidade em árvores com menores valores de CAP. Em algumas florestas tropicais não perturbadas, os períodos de instabilidade ou desequilíbrio envolvendo mortalidade e recrutamento são comuns e fazem parte de seus ciclos rítmicos, caracterizando períodos alternados de maior e menor mortalidade ou recrutamento, biomassa e densidade (GARCIA et al., 2015). Dentre os vários fatores potenciais responsáveis por esse desequilíbrio na comunidade arbórea, pode-se destacar os distúrbios como a principal causa de instabilidade em florestas tropicais (SANTOS et al., 2017). A mortalidade na menor classe diamétrica pode ter ocorrido em função de alguma perturbação natural, como por exemplo, os processos relacionados aos efeitos de borda ou estresse hídrico, uma vez que indivíduos menores são mais propensos à sazonalidade climática (SANTOS et al., 2017).

Dentre as 28 famílias botânicas inventariadas em ambos os levantamentos, Fabaceae se destacou em números de espécies. Esse resultado já era esperado, pois vários outros estudos observaram a predominância de espécies de Fabaceae (VIEIRA et al., 2014; GIÁCOMO et al., 2015). Mantovani e Martins (1993) confirmam que Fabaceae é a família mais diversificada na maioria dos levantamentos realizados no Cerrado. A elevada riqueza e densidade dessa família em florestas, sobretudo em locais alterados, podem ser em função da capacidade de muitas espécies desta família rebrotar a partir de raízes (RODRIGUES et al., 2004). Além disso, Córdula et al. (2014) corroboram afirmando que, a síndrome de dispersão predominante para esta família é a autocórica, seguida pela zoocórica, o que é um fator determinante na distribuição espacial dos indivíduos adultos no ambiente.

O padrão de "J-invertido" encontrado nos dois inventários realizados neste estudo indica um balanço positivo entre recrutamento e mortalidade, sendo característico de populações autoregenerativas. Esse padrão só ocorre quando os indivíduos menores substituem, sucessivamente, os indivíduos adultos na população, indicando que a vegetação dessa área é composta, basicamente, por indivíduos jovens em pleno desenvolvimento (SILVA JUNIOR, 2004), o que é típico de comunidades vegetais naturais estabilizadas (DURIGAN 
2009). Esse comportamento é comum às florestas inequiâneas tropicais (SOUZA et al., 2014) e inúmeros estudos já obtiveram esse padrão como resultado em suas pesquisas (OLIVEIRA et al., 2018; TRAUTENMÜLLER et al., 2019).

\section{Conclusões}

O fragmento de Cerradão, no intervalo de tempo de três anos, apresentou algumas mudanças, mas não o suficiente para alterar significativamente sua florística e sua estrutura fitossociológica. Para detecção de mudanças quanto ao recrutamento e mortalidade de espécies neste ecossistema, recomenda-se que seja estendido o período de monitoramento, com a realização de maior número de inventários.

\section{Referências}

CARVALHO, M.A.F. et al. Florística, fitossociologia e estrutura diamétrica de um remanescente florestal no município de Gurupi, Tocantins. Revista Verde de Agroecologia e Desenvolvimento Sustentável, v.11, n. 4, p. 5966, 2016.

CÓRDULA, E.; MORIM, M.P.; ALVES, M. Morfologia de frutos e sementes de Fabaceae ocorrentes em uma área prioritária para a conservação da Caatinga em Pernambuco, Brasil. Rodriguésia, v. 65, n. 2, p. 505-516, 2014.

DARONCO, C.; MELO, A. C. G.; DURIGAN, G. Ecossistema em restauração versus ecossistema de referência: estudo de caso da comunidade vegetal de mata ciliar em região de Cerrado, Assis, SP, Brasil. Hoehnea, v. 40, n. 3, p. 485-498, 2013.

DINIZ, E.S. et al. Long-term monitoring of diversity and structure of two stands of an Atlantic Tropical Forest. Biodiversity Data
Journal, v. 5, p.1-33, 2017.

DURIGAN, G. Estrutura e diversidade de comunidades florestais. In: Martins SV (ed). Ecologia de florestas tropicais do Brasil. Viçosa, UFV, pp 185-215, 2009.

FEELEY, K. J. et al. Directional changes in the species composition of a tropical forest. Ecology, v. 92, n. 4, p. 871-882, 2011.

FELFILI, J.M. et al. Changes in the floristic composition of cerrado sensu stricto in Brazil over a nine-years period. Journal of tropical Ecology, v. 16, n.4, p.579-590, 2000.

GARCIA, P.O. et al. Florestas ciliares apresentação dinâmica uniforme? In: Davide AC \& Botelho SA (Eds.) Fundamentos e métodos de proteção de ecossistemas florestais: 25 anos de experiência em matas ciliares. Lavras, Editora UFLA. p. 33-72, 2015.

GIÁCOMO, R. G. et al. Florística e Fitossociologia em Áreas de Cerradão e Mata Mesofítica na Estação Ecológica de Pirapitinga, MG. Floresta e Ambiente, v. 22, n.3, p: 287298, 2015.

HARPER, J. L. Population biology of plants. Londres: Academic Press, 1977. 892 p.

LAURANCE, S. G. W. et al. Long-term variation in Amazon forest dynamics. Journal of Vegetation Science, v. 20, p. 323-333, 2009.

LIBANO, A.M.; FELFILI, J.M. Mudanças temporais na composição florística e na diversidade de um cerrado sensu stricto do Brasil Central em um período de 18 anos (1985-2003). Acta botânica brasílica, v. 20, n. 4, p. 927-936. 2006.

MANTOVANI, W.; MARTINS, F. R. Florística do cerrado na Reserva Biológica de Moji Guaçu, SP. Acta Botânica Brasílica, v. 7, n. 1, p. 33-60, 1993. 
MUELLER-DOMBOIS, D.; ELLENBERG, H. Aims and methods of vegetation ecology. New York: John Wiley\& Sons, 1974. 547 p.

PIELOU, E.C. Species diversity and pattern diversity in the study of ecological succession. Journal Theory Biology, v. 10, n. 2, p. 370-383, 1966.

O'BRIEN, K. et al. Toward a sustainable and resilient future. In: FIELD, C.B. et al. (eds.). Managing the Risks of Extreme Events and Disasters to Advance Climate Change adaptation: A Special Report of Working Groups I and II of the Intergovernmental Panel on Climate Change (IPCC). Cambridge University Press, Cambridge and New York, p. 437-486, 2012.

OLIVEIRA, H.F. et al. Modificações Florístico-estruturais em uma Floresta Neotropical. Ciência Florestal, v. 28, n. 3, p. 888-897, 2018.

PRADO-JÚNIOR, J.A. et al. Comparação florística, estrutural e ecológica da vegetação arbórea das fitofisionomias de um remanescente urbano de cerrado. Bioscience Journal, v. 28, n. 3, p. 456-471, 2012.

REIS, G.H. et al. Tree community dynamics of a northern Minas Gerais seasonally dry forest. Revista Ceres, v. 65, n.3, p. 234-242, 2018.

RODRIGUES, R. R. et al. Tree species sprouting from root buds in a semideciduous forest affected by fires. Brazilian Archives of Biology and Technology, v. 47, n. 1, p. 127133, 2004.

SANTOS, P.F. et al. Temporal changes in tree community structure and richness in a seasonally dry tropical forest in Minas Gerais, southeastern Brazil. Bosque, v. 38, p. 537-545, 2017.

SILVA-JÚNIOR, M.C. Fitossociologia e estrutura diamétrica da mata de galeria do
Taquara, na Reserva Ecológica do IBGE, DF. Revista Árvore, v. 28, n.3, p. 419-428, 2004.

SHANNON, C. E. A mathematical theory of communication. The Bell System Technical Journal, v. 27, n. 3, p. 379-423, 1948.

SOKAL, R.R.; ROHLF, F.J. Introducción a la bioestatística. (Série de Biología Fundamental, n.5) Espanha, Reverté, Barcelona, 1984.

SOUZA, C. R. et al. Projection of diametric distribution and carbon stock of a managed forest in Manaus/AM. Floresta, v. 44, n. 3, p. 525-534, 2014.

TRAUTENMÜLLER, J.W. et al. Modelagem da estrutura diamétrica em ecótono de Floresta Ombrófila Mista e Estacional Decidual submetida a manejo florestal sustentado. BIOFIX Scientific Journal v. 4 n. 1 p. 26-34 2019.

MISSOURI BOTANICAL GARDEN. Disponível em: http://www.tropicos.org. Acesso em 20/03/2019.

VIEIRA, D.S. et al. Comparação estrutural entre floresta manejada e não manejada na comunidade Santo Antônio, Estado do Pará. Ciência Florestal, v. 24, n. 4, p. 1067-1074, 2014.

WATKINSON, A. R. Plant population dynamics. In: CRA WLEY, M. J. (Ed.). Plant ecology. 2. ed. Londres: Blackwell Scientific Publications, 1997. p. 359-400.

ZAR, J.H. Biostatistical Analysis. PrenticeHall, New Jersey, 4. ed. 1998. 